\title{
The spectral radius of subgraphs of regular graphs
}

\author{
Vladimir Nikiforov \\ Department of Mathematical Sciences, University of Memphis, \\ Memphis TN 38152, USA
}

Submitted: May 25, 2007; Accepted: Sep 30, 2007; Published: Oct 5, 2007

Mathematics Subject Classification: 05C50

\begin{abstract}
Let $\mu(G)$ and $\mu_{\min }(G)$ be the largest and smallest eigenvalues of the adjacency matrix of a graph $G$. Our main results are:

(i) Let $G$ be a regular graph of order $n$ and finite diameter $D$. If $H$ is a proper subgraph of $G$, then

$$
\mu(G)-\mu(H)>\frac{1}{n D} .
$$

(ii) If $G$ is a regular nonbipartite graph of order $n$ and finite diameter $D$, then

$$
\mu(G)+\mu_{\min }(G)>\frac{1}{n D} .
$$

Keywords: smallest eigenvalue, largest eigenvalue, diameter, connected graph, nonbipartite graph
\end{abstract}

\section{Main results}

Our notation follows [1]. Specifically, $\mu(G)$ and $\mu_{\min }(G)$ stand for the largest and smallest eigenvalues of the adjacency matrix of a graph $G$.

The aim of this note is to improve some recent results on eigenvalues of subgraphs of regular graphs. Cioabă ([2], Corollary 2.2) showed that if $G$ is a regular graph of order $n$ and $e$ is an edge of $G$ such that $G-e$ is a connected graph of diameter $D$, then

$$
\mu(G)-\mu(G-e)>\frac{1}{n D} .
$$

The approach of [3] helps improve this assertion in a natural way:

Theorem 1 Let $G$ be a regular graph of order $n$ and finite diameter $D$. If $H$ is a proper subgraph of $G$, then

$$
\mu(G)-\mu(H)>\frac{1}{n D} .
$$


Since $\mu(H) \leq \mu\left(H^{\prime}\right)$ whenever $H \subset H^{\prime}$, we may assume that $H$ is a maximal proper subgraph of $G$, that is to say, $V(H)=V(G)$ and $H$ differs from $G$ in a single edge. Thus, we can deduce Theorem 1 from the following assertion.

Theorem 2 Let $G$ be a regular graph of order $n$ and finite diameter $D$. If uv is an edge of $G$, then

$$
\mu(G)-\mu(G-u v)>\left\{\begin{array}{ll}
1 /(n D), & \text { if } G-u v \text { is connected; } \\
1 /(n-3)(D-1), & \text { otherwise. }
\end{array} .\right.
$$

Furthermore, Theorem 1 implies a result about nonbipartite graphs.

Theorem 3 If $G$ is a regular nonbipartite graph of order $n$ and finite diameter $D$, then

$$
\mu(G)+\mu_{\min }(G)>\frac{1}{n D} .
$$

Finally, we note the following more general version of the lower bound in Corollary 2.2 in [2].

Lemma 4 Let $G$ be a connected regular graph and e be an edge of $G$. If $H$ is a component of $G-e$ with $\mu(H)=\mu(G-e)$, then

$$
\mu(G)-\mu(H)>\frac{1}{\operatorname{Diam}(H)|H|} .
$$

This lemma follows easily from Theorem 2.1 of [2] and its proof is omitted.

\section{Proofs}

Proof of Theorem 2 Write $\operatorname{dist}_{F}(s, t)$ for the length of a shortest path joining two vertices $s$ and $t$ in a graph $F$. Write $d$ for the degree of $G$, let $H=G-u v$, and set $\mu=\mu(H)$.

\section{Case (a): $H$ is connected.}

Let $\mathbf{x}=\left(x_{1}, \ldots, x_{n}\right)$ be a unit eigenvector to $\mu$ and let $x_{w}$ be a maximal entry of $\mathbf{x}$; we thus have $x_{w}^{2} \geq 1 / n$. We can assume that $w \neq v$ and $w \neq u$. Indeed, if $w=v$, we see that

$$
\mu x_{v}=\sum_{v i \in E(G)} x_{i} \leq(d-1) x_{v},
$$

and so $d-\mu \geq 1$, implying (1). We have

$$
d-\mu=d \sum_{i \in V(G)} x_{i}^{2}-2 \sum_{i j \in E(G)} x_{i} x_{j}=\sum_{i j \in E(G)}\left(x_{i}-x_{j}\right)^{2}+x_{u}^{2}+x_{v}^{2} .
$$


Assume first that $\operatorname{dist}_{H}(w, u) \leq D-1$. Select a shortest path $u=u_{1}, \ldots, u_{k}=w$ joining $u$ to $w$ in $H$. We see that

$$
\begin{aligned}
d-\mu & =\sum_{i j \in E(G)}\left(x_{i}-x_{j}\right)^{2}+x_{u}^{2}+x_{v}^{2}>\sum_{i=1}^{k-1}\left(x_{u_{i}}-x_{u_{i+1}}\right)^{2}+x_{u}^{2} \\
& \geq \frac{1}{k-1}\left(x_{u_{i}}-x_{u_{i+1}}\right)^{2}+x_{u}^{2}=\frac{1}{k-1}\left(x_{w}-x_{u}\right)^{2}+x_{u}^{2} \geq \frac{1}{k} x_{w}^{2} \geq \frac{1}{n D}
\end{aligned}
$$

completing the proof.

Hereafter, we assume that $\operatorname{dist}_{H}(w, u) \geq D$ and, by symmetry, $\operatorname{dist}_{H}(w, v) \geq D$.

Let $P(u, w)$ and $P(v, w)$ be shortest paths joining $u$ and $v$ to $w$ in $G$. If $u \in P(v, w)$, then there exists a path of length at most $D-1$, joining $w$ to $u$ in $G$, and thus in $H$, a contradiction. Hence, $u \notin P(v, w)$ and, by symmetry, $v \notin P(u, w)$. Therefore, the paths $P(u, w)$ and $P(v, w)$ belong to $H$, and we have

$$
\operatorname{dist}_{H}(w, u)=\operatorname{dist}_{H}(w, v)=D .
$$

Let $Q(u, z)$ and $Q(v, z)$ be the longest subpaths of $P(u, w)$ and $P(v, w)$ having no internal vertices in common. Clearly $Q(u, z)$ and $Q(v, z)$ have the same length. Write $Q(z, w)$ for the subpath of $P(u, w)$ joining $z$ to $w$ and let

$$
Q(u, z)=u_{1}, \ldots, u_{k}, \quad Q(v, z)=v_{1}, \ldots, v_{k}, \quad Q(z, w)=w_{1}, \ldots, w_{l},
$$

where

$$
u_{1}=u, \quad u_{k}=v_{k}=w_{1}=z, \quad w_{l}=w, \quad k+l-2=D .
$$

The following argument is borrowed from [2]. Using the AM-QM inequality, we see that

$$
\begin{aligned}
d-\mu & \geq \sum_{i=1}^{k-1}\left(x_{v_{i}}-x_{v_{i+1}}\right)^{2}+x_{v}^{2}+\sum_{i=1}^{k-1}\left(x_{u_{i}}-x_{u_{i+1}}\right)^{2}+x_{u}^{2}+\sum_{i=1}^{l-1}\left(x_{w_{i}}-x_{w_{i+1}}\right)^{2} \\
& \geq \frac{2}{D-l+2} x_{z}^{2}+\frac{1}{l-1}\left(x_{w}-x_{z}\right)^{2} \geq \frac{2}{D+l-1} x_{w}^{2} \geq \frac{1}{D n}
\end{aligned}
$$

completing the proof.

\section{Case (b): $H$ is disconnected.}

Since $G$ is connected, $H$ is union of two connected graphs $H_{1}$ and $H_{2}$ such that $u \in H_{1}$, $v \in H_{2}$. Assume $\mu=\mu\left(H_{1}\right)$, set $\left|H_{1}\right|=k$ and let $\mathbf{x}=\left(x_{1}, \ldots, x_{k}\right)$ be a unit eigenvector to $\mu$. Since $d \geq 2$, we see that $\left|H_{2}\right| \geq 3$, and so, $k \leq n-3$.

Let $x_{w}$ be a maximal entry of $\mathbf{x}$; we thus have $x_{w}^{2} \geq 1 / k \geq 1 /(n-3)$. Like in the previous case, we see that $w \neq u$. Since $d \geq 2$, there is a vertex $z \in H_{2}$ such that $z \neq v$. Select a shortest path $u=u_{1}, u_{2}, \ldots, u_{l}=w$ joining $u$ to $w$ in $H_{1}$. Since $\operatorname{dist}_{G}(z, w) \leq \operatorname{diam} G=D$, we see that $l \leq D-1$. As above, we have

$$
\begin{aligned}
d-\mu & =\sum_{i j \in E(G)}\left(x_{i}-x_{j}\right)^{2}+x_{u}^{2}+x_{v}^{2}>\sum_{i=1}^{l-1}\left(x_{u_{i}}-x_{u_{i+1}}\right)^{2}+x_{u}^{2} \\
& \geq \frac{1}{l-1}\left(x_{u_{1}}-x_{u_{k}}\right)^{2}+x_{u}^{2}=\frac{1}{l-1}\left(x_{w}-x_{u}\right)^{2}+x_{u}^{2} \geq \frac{1}{l} x_{w}^{2} \geq \frac{1}{(n-3)(D-1)},
\end{aligned}
$$


completing the proof.

Proof of Theorem 3 Let $\mathbf{x}=\left(x_{1}, \ldots, x_{n}\right)$ be an eigenvector to $\mu_{\min }(G)$ and let $U=$ $\left\{u: x_{u}<0\right\}$. Write $H$ for the bipartite subgraph of $G$ containing all edges with exactly one vertex in $U$; note that $H$ is a proper subgraph of $G$ and $\mu_{\min }(H)<\mu_{\min }(G)$. Hence,

$$
\mu(G)+\mu_{\min }(G)>\mu(G)+\mu_{\min }(H)=\mu(G)-\mu(H),
$$

and the assertion follows from Theorem 1.

Acknowledgment A remark of Lingsheng Shi initiated the present note and a friendly referee helped complete it.

\section{References}

[1] B. Bollobás, Modern Graph Theory, Graduate Texts in Mathematics, 184, SpringerVerlag, New York (1998), xiv+394 pp.

[2] S. M. Cioabă, The spectral radius and the maximum degree of irregular graphs, Electronic J. Combin., 14 (2007), R38.

[3] V. Nikiforov, Revisiting two classical results on graph spectra, Electronic J. Combin., 14 (2007), R14. 